\title{
Toothpick in the porta: Recurrent liver abscesses secondary to transgastric migration of a toothpick with successful surgical exploration retrieval
}

\author{
Sami Kishawi, Mark Joseph Anderson, and Kenneth Chavin
}

\author{
Case Western Reserve University, University Hospitals Cleveland Medical Center, Cleveland, OH, USA
}

\begin{abstract}
We present a rare case of a 72-year-old man with recurrent hepatic abscesses secondary to transgastric migration of a toothpick into the liver parenchyma and left portal venous branch. Prior to identification of the foreign body, the patient received multiple courses of antibiotics and underwent image-guided catheter placement without resolution of infection. Given his refractory abdominal pain, fevers, and chills, a repeat abdominal CT was obtained and demonstrated a radio-opaque object extending through the prepyloric gastric submucosa into the liver parenchyma and left portal vein. EGD confirmed a pre-pyloric fistula tract with purulent discharge. The patient subsequently underwent exploratory laparotomy, cholecystectomy, porta hepatis exploration, removal of foreign body, and ligation of porto-enteric fistula tract. A wooden toothpick was removed in its entirety. Interval CT demonstrated resolution of hepatic abscesses and no evidence of persistent porto-enteric fistula. This exceptional case demonstrates the value of multidisciplinary care, hypervigilance for patients with refractory pyogenic liver abscesses of unknown origin, and the importance of careful preoperative planning. (Ann Hepatobiliary Pancreat Surg 2020;24:362-365)
\end{abstract}

Key Words: Toothpick; Liver abscess; Porto-enteric fistula; Foreign body; Surgery

\section{INTRODUCTION}

Pyogenic liver abscesses are the most common type of visceral abscess and are typically caused by bowel perforation, peritonitis, biliary tract disease, or hematogenous seeding from an external infected source. ${ }^{1-3}$ Foreign body ingestion is common, and most objects pass uneventfully within one week. However, an estimated one percent of ingested objects cause perforation, most commonly in the pylorus and duodenum. ${ }^{4}$ Some instances result in subacute pyogenic liver abscesses that require surgical or radiographic intervention. Given their often-delayed presentation, foreign body-induced pyogenic liver abscesses can be difficult to diagnose. We present a unique case of refractory liver abscesses caused by transmural gastric migration of an ingested toothpick into a branch of the left portal vein.

\section{CASE}

A 72-year-old man presented to the emergency department with fever, chills, and progressively-worsening right upper quadrant abdominal pain of several months' duration. He had previously been diagnosed with multiple hepatic abscesses visualized on abdominal CT and was admitted to an outside hospital for antibiotic therapy. There, he underwent repeated image-guided catheter placements in order to obtain source control. Fluid cultures repeatedly grew Actinomyces for which he was discharged home on an appropriate course of antibiotics.

Several weeks later, he presented to our hospital's emergency department. Repeat abdominal CT with IV contrast demonstrated pyogenic abscess recurrence as well as a thin, radio-opaque object traversing through the posterior gastric wall into a branch of the left portal vein (Fig. 1). Review of his prior imaging study revealed that

Received: November 14, 2019; Revised: April 19, 2020; Accepted: April 29, 2020

Corresponding author: Mark Joseph Anderson

Case Western Reserve University, University Hospitals Cleveland Medical Center, 11100 Euclid Avenue, Lakeside Building 7th Floor, Cleveland, OH 44106, USA

Tel: +1313-414-1122, E-mail: markjand16@gmail.com

Copyright (C) 2020 by The Korean Association of Hepato-Biliary-Pancreatic Surgery

This is an Open Access article distributed under the terms of the Creative Commons Attribution Non-Commercial License (http://creativecommons.org/ licenses/by-nc/4.0) which permits unrestricted non-commercial use, distribution, and reproduction in any medium, provided the original work is properly cited. Annals of Hepato-Biliary-Pancreatic Surgery • pISSN: 2508-5778 - elSSN: 2508-5859 

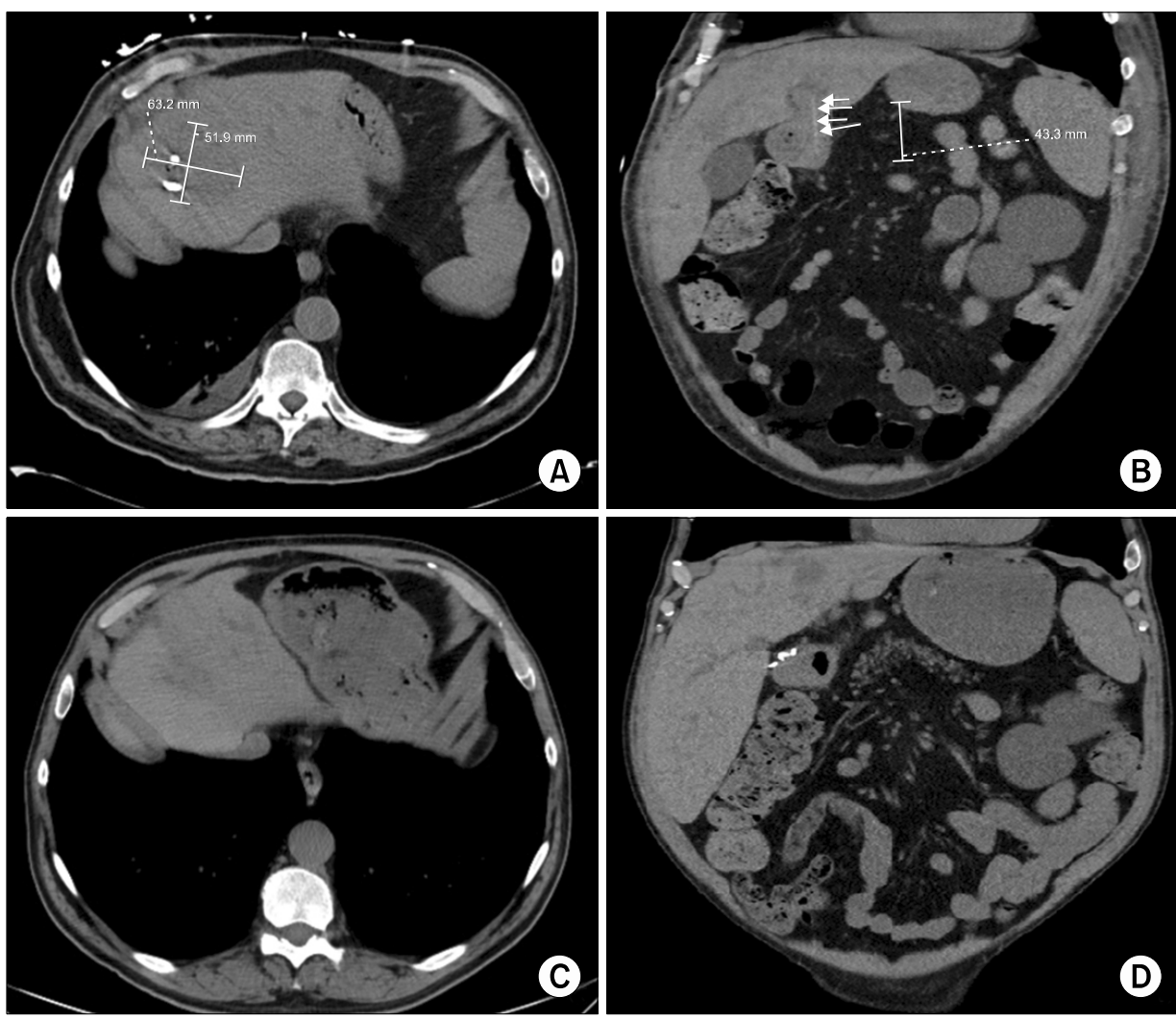

Fig. 1. CT demonstrated persistent liver abscess with IR drain in appropriate position (A). Coronal images (B) demonstrated linear, radio-opaque object extending from gastric wall to liver parenchyma and left portal venous branch. Post-operative imaging demonstrated resolution of hepatic abscess (C) and no evidence of persistent portoenteric fistula (D).
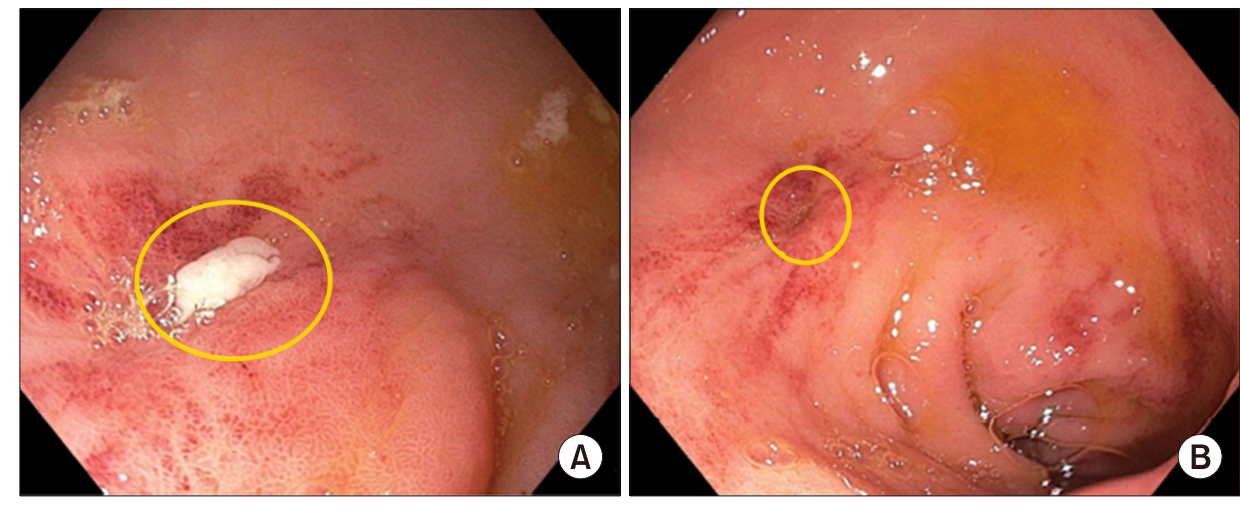

Fig. 2. Endoscopy revealed purulent output (A) from pinpoint fistulous tract $(\mathrm{B})$ in pre-pyloric gastric mucosa.

this opacity was present then, too, but was not identified in its final interpretation. The patient was admitted to the hospital and underwent EGD which revealed a pinpoint prepyloric fistula tract with purulent discharge corresponding to the location of the foreign body on CT imaging (Fig. 2).

After careful review of his imaging and thorough discussion with the patient and his family about the potential risks and benefits, the patient underwent exploratory laparotomy, cholecystectomy, porta hepatis exploration, retrieval of the foreign body, and ligation of porto-enteric fistula tract. An upper midline incision and initial explora- tion of the retrogastric space was attempted via the gastrohepatic ligament. During our dissection, thick fibrotic tissue was noted as we approached the distal lesser curve. Given the apparent sequelae of chronic abscesses near the gallbladder and chronic inflammation as we neared the fistula tract, we elected to perform a cholecystectomy and gain access the lesser sac via the foramen of Winslow. Proximal control of the portal vein was obtained and the retrogastric space was dissected bluntly with the aid of electrocautery and argon beam. Dense fibrosis limited visualization of the foreign body, therefore intraoperative ultrasound was used to locate it. Our dissection continued 

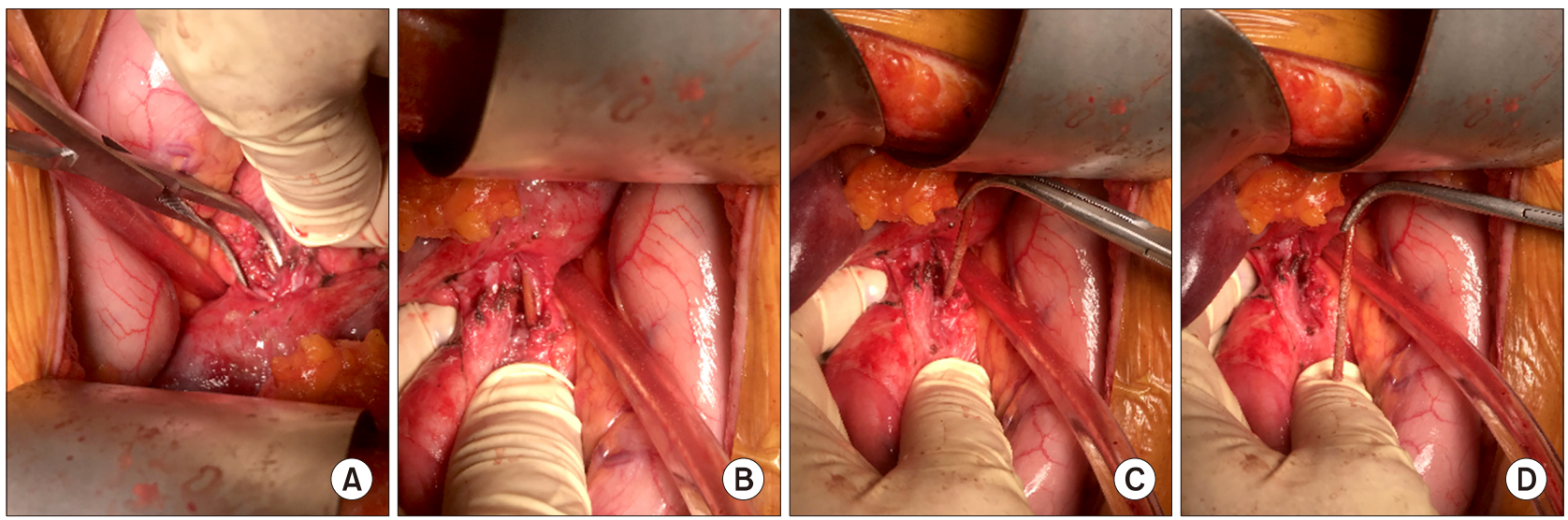

Fig. 3. Intra-operative imaging demonstrating careful dissection of porta hepatis (A) which revealed foreign body (B). A toothpick was carefully isolated (C) and retrieved (D) from the fistula tract without significant hemorrhage.

until we encountered a long, straight wooden object. A toothpick was subsequently removed from the fistula tract in its entirety and bleeding was minimal (Fig. 3). A small defect in the posterior gastric serosa was probed and then closed primarily with a figure-of-eight suture. Drains were placed in the lesser sac and near a drained abscess cavity in the gallbladder fossa.

The patient recovered well, drains were removed, and he was discharged to skilled nursing facility on an extended course of antibiotics. Neither the patient nor his family could recall when or how the toothpick was ingested. Interval outpatient $\mathrm{CT}$ demonstrated resolution of his hepatic abscesses with no evidence of persistent porto-enteric fistula or foreign body.

\section{DISCUSSION}

Pyogenic liver abscesses are relatively common and account for $13 \%$ of intra-abdominal abscesses with an annual incidence of 2.3 per 100,000 people. ${ }^{1,2}$ They are rarely caused by ingestion of a foreign object. Even more rare is their association with a porto-enteric fistula, as was witnessed in this patient case.

The first case was reported in 1898 and less than 100 cases have since been reported in the literature. The most frequently documented culprits are fish bones, toothpicks, chicken bones, and sewing needles. Patients rarely recall the instance of ingestion. ${ }^{4,5}$ Perforations commonly occur at the pylorus or duodenum where the lumen narrows or the path kinks and limits mobility of the object. Part or all of the object embeds into the liver parenchyma and acts as a nidus for infection, subsequently causing a single abscess. Given their anatomic relationships, prepyloric and pyloric perforation sites typically cause left-sided liver abscesses. Duodenal perforation sites can cause liver abscesses in the right lobe. ${ }^{6}$

What makes this case particularly unique is the foreign body's creation of a porto-enteric fistula. A thorough literature review has not revealed any previously reported cases of a foreign body causing a porto-enteric fistula. Additionally, porto-enteric fistulae are associated with a high degree of morbidity considering risk of hemorrhage. Fortunately, the fistula created by this patient's ingested toothpick formed a tight seal around the foreign body and did not cause life-threatening hemorrhage.

Diagnosis and management of pyogenic abscesses related to foreign body ingestion can be challenging due to delayed presentation, low clinical suspicion, and the infrequency of perforation. Patients are usually asymptomatic but can present with vague abdominal and nonspecific constitutional symptoms such as fever or chills. ${ }^{4,5,7,8}$ Ultrasound and CT are both useful diagnostic tools, though CT has been shown to be the most sensitive in identifying these abscesses. ${ }^{9}$ In the setting of foreign body ingestion, CT may also be helpful in ruling out other etiologies of illness and identifying the object of interest. Finally, cross sectional images aid preoperative planning and identify potential aberrant anatomy. As evidenced by this case report, small foreign bodies may be challenging to identify, and imaging must be assessed with a high degree of clinical suspicion by physicians.

Pyogenic liver abscesses can be managed in a variety 
of ways, although patient risk factors such as age, renal failure, and cirrhosis must be taken into account. Mortality rate can approach 5-6\% without considering the inherent risk of intervention. ${ }^{10}$ EGD can supplement workup and diagnosis, but potentially therapeutic intervention (i.e. foreign body retrieval) can be limited, specifically beyond the foregut. ${ }^{11}$ Percutaneous aspiration with image guidance may be preferable in many patients, especially those deemed poor surgical candidates. ${ }^{10}$ Reduction of abscess cavity size by $>50 \%$ is routinely achieved. However, source control may be impossible to obtain without removal of the inciting object. Finally, ERCP may be useful in cases related to biliary tract disease.

Surgical intervention may be required if multiple attempts at image-guided therapy have failed or foreign body retrieval is required. ${ }^{9,10}$ Open surgical retrieval may necessitate partial resection of nearby parenchymal tissue due to associated inflammatory changes. ${ }^{11}$ Surgical evaluation is also reasonable in the case of an abscess that does not respond to drainage and antibiotic therapy. ${ }^{5}$

This case demonstrates the complexities of diagnosis and management of refractory pyogenic abscesses. Although rare, a high degree of clinical suspicion is likely to expedite diagnosis and resolution of disease caused by foreign body aspiration. Thus, early surgical consultation for cases with unclear etiology is recommended. Furthermore, cross sectional imaging with IV contrast should be obtained in all appropriate patients and careful consideration of patient risk factors should be advocated prior to surgical intervention. Multidisciplinary care and a critical eye when evaluating diagnostic imaging are necessary to identify potential causes of pyogenic abscess.

\section{ORCID}

Sami Kishawi: https://orcid.org/0000-0002-2606-7225

Mark Joseph Anderson:

https://orcid.org/0000-0002-8015-7205

Kenneth Chavin: https://orcid.org/0000-0003-0818-4847

\section{REFERENCES}

1. Altemeier WA, Culbertson WR, Fullen WD, Shook CD. Intra-abdominal abscesses. Am J Surg 1973;125:70-79.

2. Kaplan GG, Gregson DB, Laupland KB. Population-based study of the epidemiology of and the risk factors for pyogenic liver abscess. Clin Gastroenterol Hepatol 2004;2:1032-1038.

3. Huang CJ, Pitt HA, Lipsett PA, Osterman FA Jr, Lillemoe KD, Cameron JL, et al. Pyogenic hepatic abscess. Changing trends over 42 years. Ann Surg 1996;223:600-607; discussion 607-609.

4. Bekki T, Fujikuni N, Tanabe K, Amano H, Noriyuki T, Nakahara M. Liver abscess caused by fish bone perforation of stomach wall treated by laparoscopic surgery: a case report. Surg Case Rep 2019;5:79.

5. Santos SA, Alberto SC, Cruz E, Pires E, Figueira T, Coimbra E, et al. Hepatic abscess induced by foreign body: case report and literature review. World J Gastroenterol 2007;13:1466-1470.

6. Leggieri N, Marques-Vidal P, Cerwenka H, Denys A, Dorta G, Moutardier V, et al. Migrated foreign body liver abscess: illustrative case report, systematic review, and proposed diagnostic algorithm. Medicine (Baltimore) 2010;89:85-95.

7. Horii K, Yamazaki O, Matsuyama M, Higaki I, Kawai S, Sakaue Y. Successful treatment of a hepatic abscess that formed secondary to fish bone penetration by percutaneous transhepatic removal of the foreign body: report of a case. Surg Today 1999;29:922-926.

8. de la Vega M, Rivero JC, Ruíz L, Suárez S. A fish bone in the liver. Lancet 2001;358:982.

9. Santos-Rosa OM, Lunardelli HS, Ribeiro-Junior MA. Pyogenic liver abscess: diagnostic and therapeutic management. Arq Bras Cir Dig 2016;29:194-197.

10. Meddings L, Myers RP, Hubbard J, Shaheen AA, Laupland KB, Dixon E, et al. A population-based study of pyogenic liver abscesses in the United States: incidence, mortality, and temporal trends. Am J Gastroenterol 2010;105:117-124.

11. Kanazawa S, Ishigaki K, Miyake T, Ishida A, Tabuchi A, Tanemoto K, et al. A granulomatous liver abscess which developed after a toothpick penetrated the gastrointestinal tract: report of a case. Surg Today 2003;33:312-314. 